\title{
Situación actual de Gentianella herrerae (Reimers) Zarucchi (Gentianaceae) en la Región Cusco, Perú
}

\section{Actual situation of Gentianella herrerae (Reimers) Zarucchi (Gentianaceae) in the Region of Cusco, Peru}

\author{
Alfredo Tupayachi Herrera \\ Investigador Asociado al Herbario (CUZ); Sociedad Botánica del Cusco (SBC).atupayachi@yahoo.com
}

\section{Resumen}

En la presente nota científica se da a conocer la situación actual de la especie hoy nominada como Gentianella herrerae (Reimers) Zarucchi colectada por única vez por el botánico cusqueño F. L. Herrera en la localidad de Jachacalla, Hacienda Ccapana Quispicanchi y enviado al herbario del Field Museum de Berlin (B) en 1926, identificada entonces como especie nueva Gentiana herrerae Raimers en 1929. Esta especie no se encuentra registrada en ningún herbario peruano (Castillo et al. 2006). En 2011 fue colectado por segunda vez por el autor de esta nota después de 85 años, lo que podrá ser considerando como un redescubrimiento de la especie.

Palabras Clave: Situación actual, F. L. Herrera, Gentiana, Gentianella, Flora, Región Cusco.

\begin{abstract}
In this scientific note we present the current situation of the species named today as Gentianella herrerae (Reimers) Zarucchi collected only once by the botanist F. L. Herrera in the town of Jachacalla, Hacienda Ccapana Quispicanchi and sent to the Herbarium of the Field Museum of Berlin (B) in 1926, then identified as a new species Gentiana herrerae Raimers in 1929. This species is not registered in any Peruvian herbarium (Castillo et al. 2006) In 2011 it was collected for the second time by the author of this note after 85 years, which may be considered as a rediscovery of the species.
\end{abstract}

KeyWord: Actual situation, F. L. Herrera, Gentiana, Gentianella. Flora, Cusco Region.

\section{Introducción}

La diversidad de la flora en los Altos Andes de la Región Cusco, entre los 3,800 y 4,800 m de altitud, está muy bien representada por especies de las familias Asteraceae, Valerianaceae, Malvaceae, Gentianaceae, Caryophyllaceae, Ranunculaceae y Poaceae entre otras, que manifiestan todo su esplendor en la antesis floral, durante la estación de lluvias (Tupayachi 2004).
La vegetación característica corresponde a un matorral abierto de arbustos bajos, dominado por un tapiz de hierbas que se esparcen a medida que ascienden la gradiente altitudinal, formando en algunos casos cojines, placas, rosetas y matas de pastos, que se enrarecen y desaparecen al llegar a la base de los glaciares, al toparse con los macizos cordilleranos o suelos desnudos producto de la desglaciación. 
En los Andes de la Región, los trabajos de prospección de la flora, formalmente se iniciaron con Augusto Weberbauer (1905), Fortunato L. Herrera (1922) y Julio César Vargas (1930); a quienes hoy en día se les considera como pioneros en las colecciones y descripciones de plantas del sur del Perú; el trabajo continúa con el esfuerzo acumulativo por botánicos cusqueños, en el marco de convenios celebrados entre la Universidad Nacional San Antonio Abad del Cusco con instituciones nacionales e internacionales.

Las sendas colecciones botánicas acumuladas, fueron y continúan siendo depositadas en el Herbario Vargas (CUZ) fundada en 1936 por Julio César Vargas Calderón y mantiene en evidencia el caudal de la riqueza florística de la región y de otras regiones vecinas; sin embargo, las colecciones anteriores a la creación del Herbario Vargas (CUZ), fueron enviadas a otros herbarios nacionales y principalmente a herbarios del exterior.

\section{Materiales y Métodos \\ Descripción de la especie}

El registro fotográfico de la especie colectada, fue enviado a Simón Pfanzelt, especialista en Biodiversidad y Evolución de las Plantas de la Universidad de Oldemburgo Alemania, quién se encontraba en Chile, justamente revisando las Gentianaceas, de quien se obtuvo respuesta inmediata con el nombre de la especie; simultáneamente se envió el material fotográfico a Susy Castillo, del Museo de Historia Natural de San Marcos que revisaba las Gentianaceas del Perú para su trabajo de pregrado, quien también confirmó la misma determinación.

\section{Descripción taxonómica}

En base a las características morfológicas de las muestras colectadas y contando con la descripción del material (Tipo: Herrera 287) depositada en el Herbario (B), se presenta a continuación, la siguiente descripción somera, acompañada de fotografías.

\section{Resultados}

La familia Gentianaceae es reconocida en el Perú por presentar alrededor de 15 géneros y aproximadamente 170 especies (Brako y Zarucchi, 1993; Ulloa et al.2004). Fortunato Luciano Herrera Garmendia (1873 - 1945), considerado como el Padre de la Botánica Nacional, exploró la cuenca alta del río Paucartambo y entre los pastizales (praderas), colectando entre otras; encontró por primera y única vez en 1926 a la especie que en ese entonces fuera identificada como Gentiana herrerae entre altitudes de 3,800 a 4,200 m que fue enviada al Field Museum of Natural History de Berlín, descrita y dedicada al colector como especie nueva, por Reimers en 1927 y publicada en Botanische Jahrbu fur Systematik Pflanzemgeschichte und Pflanzengeographic.

Macbride (1959), en Flora of Perú la cita, como Gentiana herrerae Reim, señalando como localidad de colección a Cuzco: Jachacalla, Hacienda Ccapana, Prov., Jispicanchi, entre 3,900 a 4,200 m (Herrera 287), anotando como nombre común "Phallchahuicontoy". El nuevo nombre de esta especie es Gentianella herrerae (Reimers) Zarucchi y fue dado a conocer en 1993, en una monografía de Botánica Sistemática por el Jardín Botánico de Missouri. 
Castillo, et al.(2006), indican lo siguiente:

Gentianella herrerae (Reimers) Zarucchi

Publicación: Monograf. Syst. Bot. Missouri Bot.Gard. 45: 1255. 1993.

Colección Tipo: F. Herrera 287

Herbario: (B)

Nombre Común: Pfallchahuiccontoy

Registro Departamental: Cusco

Regiones ecológicas: PSH; 3,900-4,200 m.

SINANPE: Sin registro

Estatus poblacional: CR B1a

Herbarios Peruanos: Ninguno

Hierba colectada aparentemente en la localidad sólo de la colección tipo, una planta recolectada en 1926, al Sur del Departamento del Cusco en la cuenca alta del Paucartambo.

Una segunda colecta fue realizada por A. Tupayachi, 86 años después, registrada bajo el $\mathrm{N}^{\circ}$. 8293, el 20 de octubre de 2011, colectada a aproximadamente $4 \mathrm{Km}$ al oriente del abra de Walla Walla; depositada en el Herbario Vargas (CUZ), cuyo duplicado se envió también al Herbario del Museo de Historia Natural de la Universidad Nacional Mayor de San Marcos.

Raíz bastante fuerte, fusiforme, gruesa hasta de 10 a $15 \mathrm{~cm}$, debajo de la roseta de hojas. Las hojas basales secas, pardo negruzcas, las hojas erguidas simples unidas en la base al tallo, todas ampliamente espatuladas en la base y redondeadas en el ápice, pueden medir hasta 10 a $12 \mathrm{~cm}$ de largo. coriáceas, pedúnculos florales axilares delgados desnudos y en el extremo presentan pequeñas hojas sésiles, ovadas a estrechamente oblongas hasta agudas, que protegen a numerosas flores llamativas distribuidas en cimas frondosas, erguidas que sobresalen entre las hojas; Cáliz pentámero, campanulado, sépalos unidos hasta la mitad del tubo y de ápices membranosos, con los lóbulos-
- muy agudos lanceolados. Corola pentámera aparentemente tubular, pétalos morados en los extremos y al abrirse con lóbulos, toman el color naranja - amarillento, siendo ovadas a subacutadas. Androceo con 5 estambres epipétalos y gineceo súpero. Frutos en cápsulas septicidas.

Florecen desde mediados de octubre hasta diciembre.

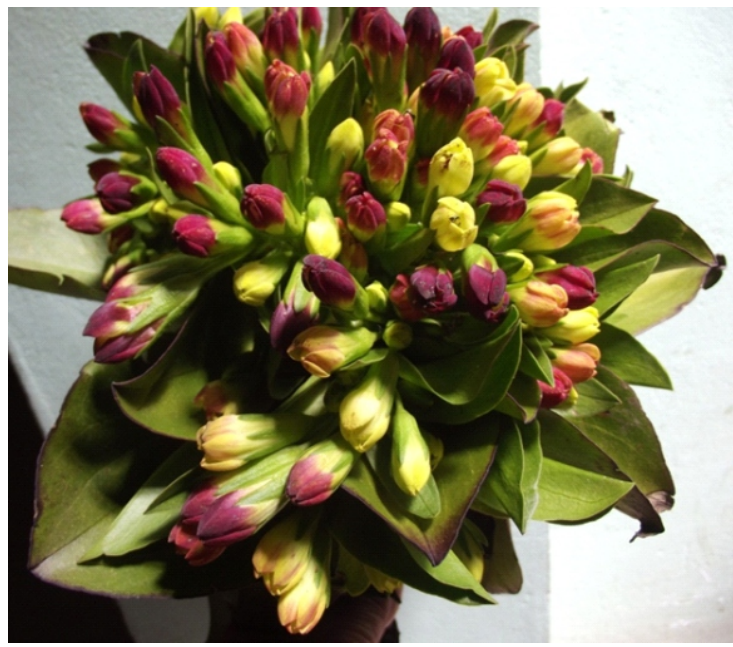

Figura 1. Gentianella herrerae (Reimers) Zarucchi (segunda colecta, A. Tupayachi $\mathrm{N}^{\circ}$. 8293)

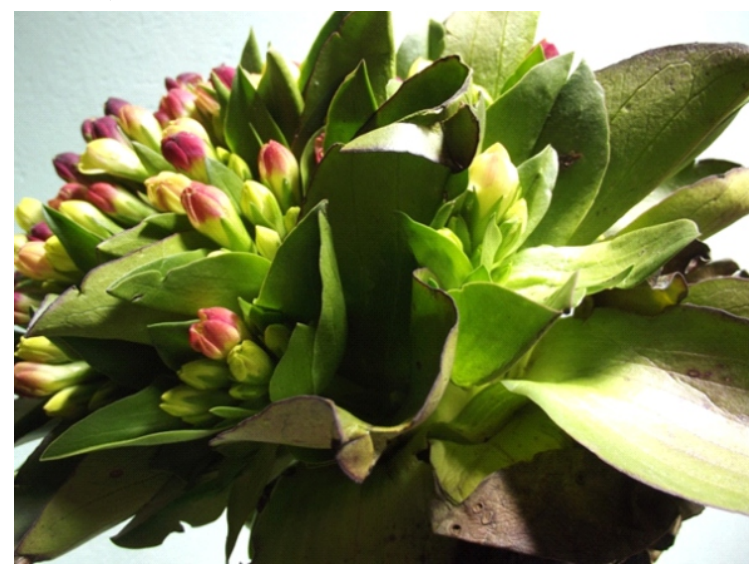

Figura 2. Gentianella herrerae en su máxima antesis floral. 
Por toda la información expuesta, estamos de acuerdo con León et al. (2006) y Castillo et al 2006), resaltando que la consideran como una especie ENDÉMICA de nuestro territorio patrio.

$\mathrm{Su}$ categorización En Peligro Crítico (CR) obedece a la definición que "cuando un taxón se enfrenta a un riesgo de extinción extremadamente alto en estado de vida silvestre, se dice que corre un peligro crítico”.

Con el subcriterio B, 1a que aborda la disminución continúa en la distribución del ecosistema, el ambiente abiótico o los procesos bióticos. Para invocar este subcriterio, la disminución debe: (i) reducir la capacidad de un ecosistema para sostener su biota nativa característica; (ii) ser de una magnitud notrivial; y (iii) ser más probable que no de continuar en el futuro (Bland et al.2016).

\section{Agradecimientos}

Expreso mi agradecimiento a Simón Pfanzelt y Susy Castillo por la determinación de la especie, igualmente a Carolina García del Herbario Nacional de la Paz - Bolivia y Hamilton Beltrán del Herbario del Museo de Historia Natural de la UNMSM, por los contactos con S. Pflanzelt y Susy Castillo respectivamente. Agradecimiento especial a José Luis Venero Gonzales colega y mejor amigo, por la revisión del manuscrito y su compromiso con la investigación de la diversidad biológica, que a pesar de los obstáculos e incomprensiones con quien continuamos en el quehacer científico.

\section{Literatura citada}

Bland, L.M., Keith, D.A., Miller, R.M., Murray, N.J. \& Rodríguez, J.P. (eds.) 2016. Directrices para la aplicación de las Categorías y Criterios de la Lista Roja de Ecosistemas de UICN, Versión 1.0. Gland, Suiza: UICN. ix + 96pp.

Brako, L. \& Zarucchi, J. L. 1993. Catalogue of the Flowering Plants and Gymnosperms in Peru. Mongr. Missouri Bot. Gard. 45.
Castillo, S., N. Salinas., B. León. \& Sánchez, I. 2006. Gentianaceas Endémicas del Perú. En: León, et al Revista peruana de Biología. Número especial 13 (2): 339-354.

León, B.,Roque, B., Pitman, N., Ulloa-Ulloa, N., Jorgensen, P. \& Cano, A. (Eds.). 2006. El Libro Rojo de las Plantas Endémicas del Perú. Revista Peruana de Biología número especial 13(2).

Macbride, J.F. 1959. Gentianaceae. Flora of Peru. Bot. Ser., Field Mus. Nat. Hist., Bot. Ser. 13 part 5(1): 270-293.

Reimers, H. J. 1929. Botanische Jahrbücher für Systematik, Pflanzengeschichte und Pflanzengeographie 62(4): 330-331.

Tupayachi, A. 2004. Evaluación de los bosques altoandinos de Polylepis (Rosaceae) del Valle Sagrado de los Inkas, para una propuesta de Área de Conservación Regional (A.C.R.). Tesis de Maestría. Universidad Nacional de San Antonio Abad del. Cusco. 238 pp.

UICN (Unión Internacional de Conservación de la Naturaleza). 2001. Categorías y Criterios de la Lista Roja de la UICN. Versión 3.1. 33 pp. The Nature Bureau, Newbury, Reino Unido.

Ulloa Ulloa, C., Zarucchi.J. \& León, B. 2004. Diez años de adiciones a la flora del Perú: 1993-2003. Arnaldoa, Ed. Especial 7-242. 